\title{
Stochastically Perturbed Chains of Variable Memory
}

\author{
Nancy L. Garcia * \\ Universidade Estadual de Campinas, Departamento de Estatística. \\ Lucas Moreira ${ }^{\dagger}$ \\ Universidade de Brasília, Departamento de Estatística.
}

August 21, 2021

\begin{abstract}
In this paper, we study inference for chains of variable order under two distinct contamination regimes. Consider we have a chain of variable memory on a finite alphabet containing zero. At each instant of time an independent coin is flipped and if it turns head a contamination occurs. In the first regime a zero is read independent of the value of the chain. In the second regime, the value of another chain of variable memory is observed instead of the original one. Our results state that the difference between the transition probabilities of the original process and the corresponding ones of the contaminated process may be bounded above uniformly. Moreover, if the contamination probability is small enough, using a version of the Context algorithm we are able to recover the context tree of the original process through a contaminated sample.
\end{abstract}

Key words: Zero-inflated processes, Process-contamination, Robust statistics, Variable length chains.

\section{Introduction}

The goal of this paper is to answer the question proposed by Collet, Galves and Leonardi (2008): "Is it possible to recover the context tree of a variable length Markov chain from a noisy sample of the chain?" In this paper, we answer positively this question for two distinct contamination models.

Unbounded variable length Markov chains define a very flexible class of stochastic chains of infinite order on a finite alphabet. The idea is that for each past, only a finite suffix of the past, called context, is enough to predict the next symbol. These suffixes can

\footnotetext{
${ }^{*}$ Rua Sérgio Buarque de Holanda, 651, CEP 13083-859, Campinas-SP, Brazil. E-mail: nancy@ime.unicamp.br

${ }^{\dagger}$ Campus Universitário Darcy Ribeiro ICC Centro - Bloco A - Asa Norte, CEP 70910-900, Brasília-DF, Brazil. E-mail: lmoreira@unb.br
} 
be represented by a countable, complete tree of finite contexts called context tree. In a probabilistic suffix tree there is a transition probability associated to each context.

Probabilistic suffix trees were first introduced by Rissanem (1983) in the finite case as a flexible and parsimonious modelization tool for data compression, approximating Markov chains of finite orders. He called his model finitely generated source. In his work, not only he introduces the model but also he proposes the algorithm Context to estimate the context needed to predict the next symbol, given a finite sample in an effective way. These models became popular in the statistics literature under the name Variable Length Markov Chains coined by Bühlmann and Wyner (1999). We refer the reader to Galves and Löcherbach (2008) for a detailed review on the subject.

In this paper we will study two contamination regimes for chains of infinite order on a finite alphabet. In the first regime, for simplicity, we assume the alphabet to be binary and at each step the symbol 1 turns into a 0 with a small fixed probability independently of everything. In the second regime, consider that we have another chain of infinite order with the same alphabet as the original one. At each instant of time the process randomly chooses the contaminant process over the original one with a small fixed probability.

Our results state that the difference between the conditional probabilities of the original process and the corresponding ones of the contaminated process may be limited above uniformly. Furthermore, we show that this upper bound is an increasing function of the contamination probability.

Using a variant of the algorithm Context presented in Galves and Leonardi (2008), our first result proves that even though a contaminated sample was used, the estimated tree recovers the context tree of the original process. That is, the proposed estimator of the context tree is robust.

We also observed that the results obtained for the first regime can be easily extended to chains taking values on a finite size alphabet.

Our paper is organized as follows, Section 2 presents some basic definitions. Section 3 presents the contamination regimes and our main results. Section 4 is dedicated to the proof of the results. Section 5 compares the results presented in this work with the corresponding one presented in Collet, Galves and Leonardi (2008).

\section{Definitions}

Without loss of generality, let us consider the alphabet $\mathcal{A}=\{0,1, \ldots, N-1\}$, with size $|\mathcal{A}|=N$.

Given two integers $m \leq n$, we denote by $a_{m}^{n}$ the string $a_{m} \ldots a_{n}$ of symbols in $A$. For any $m \leq n$, the length of the string $a_{m}^{n}$ is denoted by $l\left(a_{m}^{n}\right)$ and defined by $n-m+1$. We will often use the notation $\emptyset$ which will stand for the empty string, having length $|\emptyset|=0$. For any $n \in \mathbb{Z}$, we will use the convention that $a_{n+1}^{n}=\emptyset$, and naturally $l\left(a_{n+1}^{n}\right)=0$. Given two strings $v$ and $v^{\prime}$, we denote by $v v^{\prime}$ the string of length $l(v)+l\left(v^{\prime}\right)$ obtained by concatenating the two strings. If $v^{\prime}=\emptyset$, then $v \emptyset=\emptyset v=v$. The concatenation of strings is also extended to the case where $v=\ldots a_{-2} a_{-1}$ is a semi-infinite sequence of symbols. If $n \in\{1,2, \ldots\}$ and $v$ is a finite string of symbols in $A, v^{n}=v \ldots v$ is the concatenation of 
$n$ times the string $v$. In the case where $n=0, v^{0}$ is the empty string $\emptyset$. We say that the sequence $s$ is a suffix of the sequence $\omega$ if there exists a sequence $u$, with $l(u) \geq 1$, such that $\omega=u s$. In this case we write $s \prec \omega$. When $s \prec \omega$ or $s=\omega$ we write $s \preceq \omega$. Given a finite sequence $\omega$ we denote by $\operatorname{suf}(\omega)$ the largest suffix of $\omega$. Let

$$
\mathcal{A}_{-\infty}^{-1}=\mathcal{A}^{\{\ldots,-2,-1\}} \quad \text { and } \quad \mathcal{A}^{\star}=\bigcup_{j=0}^{+\infty} A^{\{-j, \ldots,-1\}},
$$

be, respectively, the set of all infinite strings of past symbols and the set of all finite strings of past symbols. The case $j=0$ corresponds to the empty string $\emptyset$. Finally, we denote by $\underline{a}=\ldots a_{-2} a_{-1}$ the elements of $A_{-\infty}^{-1}$.

Throughout this paper, we consider $\mathbf{X}=\left\{X_{t}, t \in \mathbb{Z}\right\}$ and $\mathbf{Y}=\left\{Y_{t}, t \in \mathbb{Z}\right\}$ stationary ergodic stochastic processes over the same finite alphabet $\mathcal{A}$. Given two sequences $\omega, v \in$ $\mathcal{A}_{-\infty}^{-1}$ and symbol $a, b \in \mathcal{A}$, let

$$
\begin{gathered}
p_{X}(a \mid \omega):=\mathbb{P}\left(X_{0}=a \mid X_{-1}=\omega_{-1}, X_{-2}=\omega_{-2}, \ldots\right), \\
p_{Y}(b \mid v):=\mathbb{P}\left(Y_{0}=b \mid Y_{-1}=v_{-1}, Y_{-2}=v_{-2}, \ldots\right),
\end{gathered}
$$

that is, the $\mathbf{X}$ and $\mathbf{Y}$ processes are compatible with the transition probabilities $p_{X}(\cdot \mid \cdot)$ and $p_{Y}(\cdot \mid \cdot)$, respectively. Given two finite sequences $\omega, v \in \mathcal{A}_{-j}^{-1}$ we denote by

$$
\mu_{X}(\omega):=\mathbb{P}\left(X_{-j}^{-1}=\omega\right), \quad \mu_{Y}(v):=\mathbb{P}\left(Y_{-j}^{-1}=v\right)
$$

the stationary probabilities of the cylinders defined by the sequences $\omega$ and $v$, respectively.

Definition 2.1. (1) Non-nullness. A process $\mathbf{X}$ is said to be non-null if it satisfies

$$
\alpha_{X}:=\inf \left\{p_{X}(a \mid \omega): a \in \mathcal{A}, \omega \in \mathcal{A}_{-\infty}^{-1}\right\}>0
$$

(2) Summable continuity rate. A process $\mathbf{X}$ has summable continuity rate if

$$
\beta_{X}:=\sum_{k \in \mathbb{N}} \beta_{k, X}<\infty
$$

where the sequence $\left\{\beta_{k, X}\right\}_{k \in \mathbb{N}}$ is defined by

$$
\beta_{k, X}:=\sup \left\{\left|1-\frac{p_{X}(a \mid \omega)}{p_{X}(a \mid v)}\right|: a \in \mathcal{A}, v, \omega \in \mathcal{A}_{-\infty}^{-1} \text { with } \omega \stackrel{k}{=} v\right\} \text {. }
$$

Here, $\omega \stackrel{k}{=} v$ means that $\omega_{-k}^{-1}=v_{-k}^{-1}$. The sequence $\left\{\beta_{k, X}\right\}_{k \in \mathbb{N}}$ is called continuity rate of the process $\mathbf{X}$.

Definition 2.2. A sequence $\omega \in \mathcal{A}_{-j}^{-1}$ is a context for the process $\mathbf{X}$ if it satisfies 
(1) For all semi-infinite sequence $x_{-\infty}^{-1}$ having $\omega$ as a suffix,

$$
\mathbb{P}\left(X_{0}=a \mid X_{-\infty}^{-1}=x_{-\infty}^{-1}\right)=p_{X}(a \mid \omega), \text { for all } a \in \mathcal{A} .
$$

(2) No suffix of $\omega$ satisfies (2.1).

An infinite context is a semi-infinite sequence $\omega_{-\infty}^{-1}$ such that none of its suffixes $\omega_{-j}^{-1}$, $j=1,2, \ldots$ is a context.

It is easy to see that the set of all contexts (finite or infinite) can be identified with the set of leaves of a rooted tree with a countable set of finite labeled branches. This tree is called context tree of the process $\mathbf{X}$ and it will be denoted by $\mathcal{T}_{X}$.

Definition 2.3. Given an integer $K$, define the tree $\mathcal{T}_{X}$ truncated at level $K$ by

$$
\left.\mathcal{T}_{X}\right|_{K}:=\left\{\omega \in \mathcal{T}_{X}: l(\omega) \leq K\right\} \cup\left\{\omega: l(\omega)=K \text { and } \omega \prec u \text {, for some } u \in \mathcal{T}_{X}\right\} .
$$

Given an integer $k \geq 1$, define

$$
\mathcal{C}_{k}:=\left\{\left.u \in \mathcal{T}_{X}\right|_{k}: p_{X}(a \mid u) \neq p_{X}(a \mid \operatorname{suf}(u)), \text { for some a } \in \mathcal{A}\right\}
$$

and

$$
D_{k}:=\min _{u \in C_{k}} \max _{a \in \mathcal{A}}\left\{\left|p_{X}(a \mid u)-p_{X}(a \mid \operatorname{suf}(u))\right|\right\} .
$$

From the definition, we can see that $D_{k}>0$ for all $k \geq 1$.

Algorithm Context Generically, let $\mathbf{Z}$ be an infinite order process on the alphabet $\mathcal{A}$. We assume that $\mathbf{Z}$ is compatible with a transition kernel $p_{Z}(\cdot \mid \cdot)$. Later, the process $\mathbf{Z}$ will denote both contamination models studied in this work. Let $Z_{1}, Z_{2}, \ldots, Z_{n}$ be a random sample of the process $\mathbf{Z}$. For any finite sequence $\omega$, with $l(\omega) \leq n$, we denote by $N_{n}(\omega)$ the number of occurrences of $\omega$ in the sample, that is

$$
N_{n}(\omega)=\sum_{t=0}^{n-l(\omega)} \mathbf{1}_{\left\{Z_{t+1}^{t+l(\omega)}=\omega\right\}} .
$$

For any element $a \in \mathcal{A}$ and for any finite sequence $\omega$, the empirical transition kernel $\hat{p}_{Z}(a \mid \omega)_{n}$ is defined by

$$
\hat{p}_{Z}(a \mid \omega)_{n}=\frac{N_{n}(\omega a)+1}{N_{n}(\omega \cdot)+|\mathcal{A}|}, \quad \text { where } \quad N_{n}(\omega \cdot)=\sum_{b \in \mathcal{A}} N_{n}(\omega b) .
$$

A modification of Rissanen's context tree estimator proposed in Galves and Leonardi (2008) which we will use in this work is given below. First, let us define the operator

$$
\Delta_{n}(\omega):=\max _{a \in \mathcal{A}}\left|\hat{p}_{Z}(a \mid \omega)_{n}-\hat{p}_{Z}(a \mid \operatorname{suf}(\omega))_{n}\right|
$$

for any finite sequence $\omega$.

Definition 2.4. (Galves and Leonardi, 2008). For any $\delta>0$ and $d<n$, the context tree estimator $\hat{\mathcal{T}}_{n}^{\delta, d}$ is the set containing all sequences $\omega \in \mathcal{A}_{-d}^{-1}$, such that $\Delta_{n}($ asuf $(\omega))>\delta$ for some $a \in \mathcal{A}$ and $\Delta_{n}(u \omega) \leq \delta$ for any $u \in \mathcal{A}_{-d}^{-l(\omega)}$. 


\section{Contamination Regimes and Results}

\subsection{Zero inflated contamination}

Initially, we consider $\mathbf{X}$ a stationary process compatible with $p_{X}(\cdot \mid \cdot)$ but taking values in the binary alphabet $\mathcal{A}=\{0,1\}$. Let $\boldsymbol{\xi}=\left\{\xi_{t}, t \in \mathbb{Z}\right\}$ be a sequence of i.i.d. Bernoulli random variables taking values on $\{0,1\}$, independent of the process $\mathbf{X}$, with

$$
\mathbb{P}\left(\xi_{t}=1\right)=1-\varepsilon,
$$

where $\varepsilon$ is a noise parameter fixed in $(0,1)$.

We define the Zero inflated contamination model by

$$
Z_{t}:=X_{t} \cdot \xi_{t}, \quad t \in \mathbb{Z}
$$

It is easy to see that $\mathbf{Z}$ will be an infinite order process even if the process $\mathbf{X}$ is a Markov chain of order one.

Theorem 3.1. If $\mathbf{X}$ is non-null and has summable continuity rate and $\mathbf{Z}$ is defined by (3.1) then, for any $\varepsilon \in(0,1)$, we have

$$
\sup _{k \geq 1} \sup _{\omega_{-k}^{-1} \in \mathcal{A}_{-k}^{-1}} \sup _{a \in \mathcal{A}}\left|p_{Z}\left(a \mid \omega_{-k}^{-1}\right)-p_{X}\left(a \mid \omega_{-k}^{-1}\right)\right| \leq \varepsilon\left[1+\frac{4 \beta_{X}}{\min \left(1,(1+\varepsilon) \alpha_{X} \beta_{X}^{*}\right)}\right],
$$

where $\beta_{X}^{*}=\prod_{k=0}^{+\infty}\left(1-\beta_{k, X}\right)>0$.

In order to recover the truncated context tree process $\mathbf{X}$ by using a sample of the perturbed process $\mathbf{Z}$, we establish the second result of this section.

Theorem 3.2. Let $K$ be an integer and consider $Z_{1}, Z_{2}, \ldots, Z_{n}$ a random sample of the perturbed process $\mathbf{Z}$. Then, there exists a constant $c_{1}$ and an integer $d$ depending on the process $\mathbf{X}$ such that for any $\epsilon \in\left(0, D_{d} / 2 c_{1}\right)$, any $\delta \in\left(c_{1} \epsilon, D_{d}-c_{1} \epsilon\right)$, there exists $n_{0}(\delta)$ such that for any $n>n_{0}$ we have

$$
\mathbb{P}\left(\left.\hat{\mathcal{T}}_{n}^{\delta, d}\right|_{K} \neq\left.\mathcal{T}_{X}\right|_{K}\right) \leq c_{2} \exp \left\{-c_{3}(n-d)\right\},
$$

where $\hat{\mathcal{T}}_{n}^{\delta, d}$ is as in Definition 2.4. The constants are explicit and given by

$$
\begin{aligned}
& \text { (1) } c_{1}=2\left[1+\frac{4 \beta_{X}}{\min \left(\alpha_{X} \beta_{X}^{*}, 1\right)}\right], c_{2}=2^{d} 12 e^{\frac{1}{e}}, c_{3}=\frac{\left[\min \left(D_{d}-\delta, \delta\right)-2 \bar{k}\right]^{2} \alpha_{X}^{2 d}(1-\varepsilon)^{3 d+1}}{256 e(d+1)\left(1+\frac{\beta_{X}}{\alpha_{X}}\right)}, \\
& \text { (2) } d=\max _{u \notin \mathcal{T}_{X}, l(u)<K} \min \left\{k: \text { there exists } \omega \in \mathcal{C}_{k} \text { with } u \prec \omega\right\}, \\
& \text { (3) } n_{0}=\frac{6}{\left\{D_{d}-\delta-2 \varepsilon\left[1+\frac{4 \beta_{X}}{\min \left(1,(1+\varepsilon) \alpha_{X} \beta_{X}^{*}\right)}\right]\right\} \alpha_{X}^{d}(1-\varepsilon)^{d}}+d, \\
& \text { (4) } \bar{k}=\varepsilon\left[1+\frac{4 \beta_{X}}{\min \left(1,(1+\varepsilon) \alpha_{X} \beta^{*}\right)}\right]+\frac{3}{(n-d) \alpha_{X}^{d}(1-\varepsilon)^{d}} .
\end{aligned}
$$

Corollary 3.3. For all integer $K$ and for almost all infinite sample $Z_{1}, Z_{2}, \ldots$ there exists an $\bar{n}$ such that, for any $n \geq \bar{n}$ we have

$$
\left.\hat{\mathcal{T}}_{n}^{\delta, d}\right|_{K}=\left.\mathcal{T}_{X}\right|_{K}
$$

where $d$ and $\delta$ are chosen as in Theorem 3.2. 


\subsection{Process contamination}

Let $\mathbf{X}$ and $\mathbf{Y}$ be independent processes taking values on the alphabet $\mathcal{A}=\{0,1, \ldots, N-1\}$ and compatible with the transition probabilities $p_{X}(\cdot \mid \cdot)$ and $p_{Y}(\cdot \mid \cdot)$ respectively. Furthermore, we suppose that these processes are non-null and have summable continuity rate with constantes $\alpha_{X}, \beta_{X}, \alpha_{Y}$ and $\beta_{Y}$, respectively. Let $\boldsymbol{\xi}=\left\{\xi_{t}, t \in \mathbb{Z}\right\}$ be a sequence of i.i.d. random variables taking values on $\{0,1\}$, independent of the processes $\mathbf{X}$ and $\mathbf{Y}$, with

$$
\mathbb{P}\left(\xi_{t}=1\right)=1-\varepsilon,
$$

where $\varepsilon$ is a parameter fixed in $(0,1)$. Define the process $\mathbf{Z}$ by

$$
Z_{t}=\left\{\begin{array}{cc}
X_{t}, & \text { if } \xi_{t}=1 \\
Y_{t}, & \text { if } \xi_{t}=0
\end{array}\right.
$$

for all $t \in \mathbb{Z}$. In fact, the contamination we propose is that at each time the process $\mathbf{Z}$ chooses with probability $1-\epsilon$ and $\epsilon$ to use the transition law of $\mathbf{X}$ or $\mathbf{Y}$ independently of everything. Therefore, model (3.3) corresponds to

$$
p_{Z}(\cdot \mid \omega)= \begin{cases}p_{X}(\cdot \mid \omega), & \text { if } \xi_{t}=1 \\ p_{Y}(\cdot \mid \omega), & \text { if } \xi_{t}=0\end{cases}
$$

Generically, $\mathbf{Z}$ is an infinite order process and it can be interpreted as a stochastic perturbation of the process $\mathbf{X}$ if $\varepsilon$ is sufficiently small. For this model, we obtained similar results to the zero inflated contamination.

Theorem 3.4. Let $\mathbf{X}$ and $\mathbf{Y}$ be independent processes which are non-null and have summable continuity rate and $\mathbf{Z}$ defined by (3.3). Then, for all $\varepsilon \in(0,1)$, we have

$$
\sup _{k \geq 1} \sup _{a \in \mathcal{A}} \sup _{\omega_{-k}^{-1} \in \mathcal{A}_{-k}^{-1}}\left|p_{Z}\left(a \mid \omega_{-k}^{-1}\right)-p_{X}\left(a \mid \omega_{-k}^{-1}\right)\right| \leq \varepsilon\left[2+\frac{4(N-1) \beta_{X}}{\min \left(1, \alpha \beta_{\text {min }}^{*}\right)}\right],
$$

where $\beta_{X}^{*}=\prod_{k=0}^{+\infty}\left(1-\beta_{k, X}\right)>0$ and $\alpha \beta_{\min }^{*}=\min \left\{\alpha_{X} \beta_{X}^{*}, \alpha_{Y}\right\}$.

Theorem 3.5. Let $K$ be an integer and let $Z_{1}, Z_{2}, \ldots, Z_{n}$ be a random sample of the process $\mathbf{Z}$. Then, there exists a constant $c_{1}$ depending on the processes $\mathbf{X}$ and $\mathbf{Y}$ and there exists an integer $d$ depending on the process $\mathbf{X}$ such that for any $\epsilon \in\left(0, D_{d} / 2 c_{1}\right)$, any $\delta \in\left(c_{1} \epsilon, D_{d}-c_{1} \epsilon\right)$, there exists $n_{0}(\delta)$ such that for all $n>n_{0}$ we have

$$
\mathbb{P}\left(\left.\hat{\mathcal{T}}_{n}^{\delta, d}\right|_{K} \neq\left.\mathcal{T}_{X}\right|_{K}\right) \leq c_{2} \exp \left\{-c_{3}(n-d)\right\}
$$

where $\hat{\mathcal{T}}_{n}^{\delta, d}$ is as in Definition 2.4. All the constants above are explicit and given by 
(1) $c_{1}=4\left[1+\frac{2(N-1) \beta_{X}}{\min \left(\left(\alpha \beta^{*}\right) \min , 1\right)}\right], c_{2}=48 N^{d}(N+1) e^{\frac{1}{e}}, c_{3}=\frac{\left[\min \left(D_{d}-\delta, \delta\right)-2 \bar{k}\right]^{2} \alpha^{2 d}}{128 N^{2} e(d+1) \beta_{\alpha, \max }}$,

(2) $d=\max _{u \notin \mathcal{T}_{X}, l(u)<K} \min \left\{k:\right.$ there exists $\omega \in \mathcal{C}_{k}$ with $\left.u \prec \omega\right\}$,

(3) $n_{0}=\frac{2(N+1)}{\left\{D_{d}-\delta-4 \varepsilon\left[1+\frac{2(N-1) \beta_{X}}{\min \left(1,\left(\alpha \beta^{*}\right)_{\text {min }}\right)}\right]\right\} \alpha_{\text {min }}^{d}}+d, \bar{k}=\frac{\varepsilon c_{1}}{2}+\frac{N+1}{(n-d) \alpha_{\text {min }}^{d}}$,

(4) $\alpha_{\min }=\min \left\{\alpha_{X}, \alpha_{Y}\right\}, \beta_{\alpha, \max }=\min \left\{\left(1+\frac{\beta_{X}}{\alpha_{X}}\right),\left(1+\frac{\beta_{Y}}{\alpha_{Y}}\right)\right\}$.

Corollary 3.6. For all integer $K$ and for almost all infinite sample $Z_{1}, Z_{2}, \ldots$ there exists an $\bar{n}$ such that for all $n \geq \bar{n}$ we have

$$
\left.\hat{\mathcal{T}}_{n}^{\delta, d}\right|_{K}=\left.\mathcal{T}_{X}\right|_{K},
$$

where $d$ and $\delta$ are the same as in Theorem 3.5.

Robustness In Theorems 3.2 and 3.5 as well as in Corollaries 3.3 and 3.6 we can see that the estimator $\left.\hat{\mathcal{T}}_{n}^{\delta, d}\right|_{K}$ is robust. Here robustness means that even if the estimation process is based on a random sample of the perturbed process $\mathbf{Z}$, the estimator $\left.\hat{\mathcal{T}}_{n}^{\delta, d}\right|_{K}$ is able to recover the truncated context tree $\left.\mathcal{T}_{X}\right|_{K}$ of the original process $\mathbf{X}$.

\section{Proofs}

\subsection{Proof of Theorem 3.1}

In order to prove the Theorem 3.1 we establish three lemmas.

Lemma 4.1. For any $\varepsilon \in(0,1)$, any $\omega_{-\infty}^{0} \in \mathcal{A}_{-\infty}^{-1}$, any $k>j \geq 0$ and any $a, b \in \mathcal{A}$, we have

$\left|\mathbb{P}\left(X_{0}=\omega_{0} \mid X_{-j}^{-1}=\omega_{-j}^{-1}, X_{-j-1}=a, Z_{-j-1}=b, Z_{-k}^{-j-2}=\omega_{-k}^{-j-2}\right)-p_{X}\left(\omega_{0} \mid \omega_{-\infty}^{-1}\right)\right| \leq \beta_{j, X}$.

Proof. For all $j \geq 0$, we can write the following identity

$$
\begin{gathered}
\mathbb{P}\left(X_{0}=\omega_{0} \mid X_{-j}^{-1}=\omega_{-j}^{-1}, X_{-j-1}=a, Z_{-j-1}=b, Z_{-k}^{-j-2}=\omega_{-k}^{-j-2}\right) \\
=\frac{\sum_{-k} p_{X}\left(u_{-k}^{-j-2} a \omega_{-j}^{-1} \omega_{0}\right) \mathbb{P}\left(Z_{-k}^{-j-1}=\omega_{-k}^{-j-2} b \mid X_{-k}^{-j-1}=u_{-k}^{-j-2} a\right)}{\sum_{u_{-k}^{-j-2}} p_{X}\left(u_{-k}^{-j-2} a \omega_{-j}^{-1}\right) \mathbb{P}\left(Z_{-k}^{-j-1}=\omega_{-k}^{-j-2} b \mid X_{-k}^{-j-1}=u_{-k}^{-j-2} a\right)},
\end{gathered}
$$

where the last two sums are over all sequences $u_{-k}^{-j-2} \in \mathcal{A}_{-k}^{-j-2}$ such that

$$
\mathbb{P}\left(X_{-k}^{-j-1}=u_{-k}^{-j-2} a, Z_{-k}^{-j-1}=\omega_{-k}^{-j-1} b\right) \neq 0 .
$$


Now, as in Fernandez and Galves (2002), we have

$$
\inf _{v_{-\infty}^{-j-1}} p_{X}\left(\omega_{0} \mid v_{-\infty}^{-j-1} \omega_{-j}^{-1}\right) \leq p_{X}\left(\omega_{0} \mid u_{-k}^{-j-2} a \omega_{-j}^{-1}\right) \leq \sup _{v_{-\infty}^{-j-1}} p_{X}\left(\omega_{0} \mid v_{-\infty}^{-j-1} \omega_{-j}^{-1}\right) .
$$

Since the process $\mathbf{X}$ is continuous, by (4.2), it follows that

$$
p_{X}\left(\omega_{0} \mid \omega_{-\infty}^{-1}\right)-\beta_{j, X} \leq p_{X}\left(\omega_{0} \mid u_{-k}^{-j-2} a \omega_{-j}^{-1}\right) \leq p_{X}\left(\omega_{0} \mid \omega_{-\infty}^{-1}\right)+\beta_{j, X} .
$$

Thus, by plugging (4.1) into (4.3), we conclude the proof of this lemma.

Lemma 4.2. For any $\varepsilon \in(0,1)$, any $k \geq 0$ and any $\omega_{-k}^{-1} \in \mathcal{A}_{-k}^{-1}, \omega_{0} \in \mathcal{A}$, we have

$$
p_{Z}\left(\omega_{0} \mid \omega_{-k}^{-1}\right) \geq(1-\varepsilon) \alpha_{X}
$$

and

$$
\mathbb{P}\left(X_{0}=\omega_{0} \mid Z_{-k}^{-1}=\omega_{-k}^{-1}\right) \geq \alpha_{X} .
$$

Furthermore, for any $0 \leq j \leq k$ we can write

$$
\mathbb{P}\left(X_{-j-1}=\omega_{-j-1} \mid X_{-j}^{-1}=\omega_{-j}^{-1}, Z_{-k}^{-j-2}=\omega_{-k}^{-j-2}\right) \geq \alpha_{X} \beta_{X}^{*} .
$$

Proof. Initially, we will prove that (4.4) follows from (4.5). To see that, let us first consider the case $\omega_{0}=0$, we have

$$
\begin{aligned}
p_{Z}\left(0 \mid \omega_{-k}^{-1}\right)= & \varepsilon \mathbb{P}\left(X_{0}=0 \mid Z_{-k}^{-1}=\omega_{-k}^{-1}\right)+(1-\varepsilon) \mathbb{P}\left(X_{0}=0 \mid Z_{-k}^{-1}=\omega_{-k}^{-1}\right)+ \\
& +\varepsilon \mathbb{P}\left(X_{0}=1 \mid Z_{-k}^{-1}=\omega_{-k}^{-1}\right) \geq(1+\varepsilon) \alpha_{X} .
\end{aligned}
$$

Similarly, for the case $\omega_{0}=1$, we can write

$$
p_{Z}\left(1 \mid \omega_{-k}^{-1}\right)=\mathbb{P}\left(X_{0}=1, \xi_{0}=1 \mid Z_{-k}^{-1}=\omega_{-k}^{-1}\right) \geq(1-\varepsilon) \alpha_{X} .
$$

Therefore, for any $\omega_{0} \in \mathcal{A}$ we obtain $p_{Z}\left(\omega_{0} \mid \omega_{-k}^{-1}\right) \geq(1-\varepsilon) \alpha_{X}$. Now, it is easy to see that

$$
\begin{aligned}
& \mathbb{P}\left(X_{0}=\omega_{0} \mid Z_{-k}^{-1}=\omega_{-k}^{-1}\right) \\
& =\lim _{l \rightarrow+\infty} \frac{\sum_{-l}^{-1} p_{X}\left(\omega_{0} \mid \omega_{-\infty}^{-l-1} u_{-l}^{-1}\right) p_{X}\left(u_{-l}^{-1} \mid \omega_{-\infty}^{-l-1}\right) \mathbb{P}\left(\bigcap_{-k \leq-t \leq-1: u_{-t} \neq 0} \xi_{-t}=w_{-t}\right)}{\sum_{u_{-l}^{-1}} p_{X}\left(u_{-l}^{-1} \mid \omega_{-\infty}^{l-1}\right) \mathbb{P}\left(\bigcap_{-k \leq-t \leq-1: u_{-t} \neq 0} \xi_{-t}=w_{-t}\right)},
\end{aligned}
$$

where the last sums are over all the sequences $u_{-l}^{-1} \in \mathcal{A}_{-l}^{-1}$ such that

$$
\mathbb{P}\left(X_{-l}^{-1}=u_{-l}^{-1}, Z_{-k}^{-1}=\omega_{-k}^{-1}\right) \neq 0 .
$$


From the non-nullness hypothesis of the process $\mathbf{X}$, we can write $p_{X}\left(\omega_{0} \mid \omega_{-\infty}^{-l-1} u_{-l}^{-1}\right) \geq \alpha_{X}$. Consequently,

$$
\mathbb{P}\left(X_{0}=\omega_{0} \mid Z_{-k}^{-1}=\omega_{-k}^{-1}\right) \geq \alpha_{X}
$$

In order to show (4.6) we observe that the following equality is true

$$
\begin{aligned}
\mathbb{P}\left(X_{-j-1}=\omega_{-j-1} \mid X_{-j}^{-1}=\omega_{-j}^{-1}, Z_{-k}^{-j-2}=\omega_{-k}^{-j-2}\right) & \sum_{-k-2} \mathbb{P}\left(Z_{-k}^{-j-2}=\omega_{-k}^{-j-2} \mid X_{-k}^{-j-2}=x_{-k}^{-j-2}\right) p_{X}\left(x_{-k}^{-j-2} \omega_{-j-1}^{-1}\right) \\
= & \frac{x_{-k}^{-j-2}}{\sum_{x_{-k}^{-j-2}} \mathbb{P}\left(Z_{-k}^{-j-2}=\omega_{-k}^{-j-2} \mid X_{-k}^{-j-2}=x_{-k}^{-j-2}\right) p_{X}\left(x_{-k}^{-j-2} \omega_{-j}^{-1}\right)},
\end{aligned}
$$

where the the last sums are over all sequences $x_{-k}^{-j-2} \in \mathcal{A}_{-k}^{-j-2}$ such that

$$
\mathbb{P}\left(X_{-k}^{-j-2}=x_{-k}^{-j-2}, Z_{-k}^{-j-2}=\omega_{-k}^{-j-2}\right) \neq 0 .
$$

Now we can see that

$$
\frac{p_{X}\left(x_{-k}^{-j-2} \omega_{-j-1}^{-1}\right)}{p_{X}\left(x_{-k}^{-j-2} \omega_{-j}^{-1}\right)} \geq \alpha_{X} \beta_{X}^{*}
$$

The proof of the third statement of this lemma follows from the last inequality and identity (4.7).

Lemma 4.3. For any $\varepsilon \in(0,1), k>j \geq 0$ and $\omega_{-k}^{0} \in \mathcal{A}$, we have

$$
\sup _{j, k} \sup _{\omega_{-k}^{0}} \mathbb{P}\left(X_{-j-1}=\omega_{-j-1}^{\prime} \mid X_{-j}^{-1}=\omega_{-j}^{-1}, Z_{-k}^{-j-1}=\omega_{-k}^{-j-1}\right) \leq \frac{\varepsilon}{(1+\varepsilon) \alpha_{X} \beta_{X}^{*}},
$$

where $\omega_{-j-1}^{\prime} \neq \omega_{-j-1}$.

Proof. For $\omega_{-j-1}^{\prime} \neq \omega_{-j-1}$, we have

$$
\begin{aligned}
\mathbb{P}\left(X_{-j-1}=\omega_{-j-1}^{\prime} \mid X_{-j}^{-1}=\omega_{-j}^{-1}, Z_{-k}^{-j-1}=\omega_{-k}^{-j-1}\right) \\
=\frac{\mathbb{P}\left(X_{-j-1}=\omega_{-j-1}^{\prime}, Z_{-j-1}=\omega_{-j-1} \mid X_{-j}^{-1}=\omega_{-j}^{-1}, Z_{-k}^{-j-2}=\omega_{-k}^{-j-2}\right)}{\mathbb{P}\left(Z_{-j-1}=\omega_{-j-1} \mid X_{-j}^{-1}=\omega_{-j}^{-1}, Z_{-k}^{-j-2}=\omega_{-k}^{-j-2}\right)} .
\end{aligned}
$$

Now, by Lemma 4.2 and the last equality we can obtain

$$
\mathbb{P}\left(X_{-j-1}=\omega_{-j-1}^{\prime} \mid X_{-j}^{-1}=\omega_{-j}^{-1}, Z_{-k}^{-j-1}=\omega_{-k}^{-j-1}\right) \leq \frac{\varepsilon}{(1+\varepsilon) \alpha_{X} \beta_{X}^{*}},
$$

as desired. 
Proof of Theorem 3.1. For any $a \in \mathcal{A}$ and every sequence $\omega_{-k}^{-1} \in \mathcal{A}_{-k}^{-1}$, one can see that

$$
\left|p_{Z}\left(a \mid \omega_{-k}^{-1}\right)-\mathbb{P}\left(X_{0}=a \mid Z_{-k}^{-1}=\omega_{-k}^{-1}\right)\right| \leq \varepsilon .
$$

Observe that, for $k=0$, the assertion of Theorem 3.1 is trivially valid. So it remains to prove (3.2) for $k \geq 1$. In this case, we can write

$$
\begin{aligned}
\mathbb{P}\left(X_{0}=a \mid Z_{-k}^{-1}=\omega_{-k}^{-1}\right)-\mathbb{P}\left(X_{0}=a \mid X_{-k}^{-1}=\omega_{-k}^{-1}\right) \\
=\sum_{j=0}^{k-1}\left[\mathbb{P}\left(X_{0}=a \mid X_{-j}^{-1}=\omega_{-j}^{-1}, Z_{-k}^{-j-1}=\omega_{-k}^{-j-1}\right)\right. \\
\left.\quad-\mathbb{P}\left(X_{0}=a \mid X_{-j-1}^{-1}=\omega_{-j-1}^{-1}, Z_{-k}^{-j-2}=\omega_{-k}^{-j-2}\right)\right] .
\end{aligned}
$$

We can show that each parcel of the last sum can be rewritten as follows

$$
\begin{aligned}
& \sum_{b \in\{0,1\}}\left[\mathbb{P}\left(X_{0}=a \mid X_{-j}^{-1}=\omega_{-j}^{-1}, X_{-j-1}=b, Z_{-k}^{-j-1}=\omega_{-k}^{-j-1}\right)\right. \\
& \left.-\mathbb{P}\left(X_{0}=a \mid X_{-j-1}^{-1}=\omega_{-j-1}^{-1}, Z_{-k}^{-j-2}=\omega_{-k}^{-j-2}\right)\right] \\
& \quad \times \mathbb{P}\left(X_{-j-1}=b \mid X_{-j}^{-1}=\omega_{-j}^{-1}, Z_{-k}^{-j-1}=\omega_{-k}^{-j-1}\right) .
\end{aligned}
$$

For each $j, 0 \leq j \leq k-1$, the sum above has two parcels. From Lemma 4.3 the parcel corresponding to $b=\omega_{-j-1}^{\prime}$, with $\omega_{-j-1}^{\prime} \neq \omega_{-j-1}$, can be upper bounded by

$$
2 \beta_{j, X} \frac{\varepsilon}{(1+\varepsilon) \alpha_{X} \beta_{X}^{*}} .
$$

Now, for each $j$, we shall limit the parcel of (4.11) corresponding to $b=\omega_{-j-1}$ in (4.11) by

$$
\begin{aligned}
& \sum_{c \in\{0,1\}} \mid \mathbb{P}\left(X_{0}=a \mid X_{-j}^{-1}=\omega_{-j}^{-1}, X_{-j-1}=\omega_{-j-1}, Z_{-k}^{-j-1}=\omega_{-k}^{-j-1}\right) \\
& \quad-\mathbb{P}\left(X_{0}=a \mid X_{-j-1}^{-1}=\omega_{-j-1}^{-1}, Z_{-k}^{-j-2}=\omega_{-k}^{-j-2}, Z_{-j-1}=c\right) \mid \\
& \quad \times \mathbb{P}\left(X_{-j-1}=\omega_{-j-1} \mid X_{-j}^{-1}=\omega_{-j}^{-1}, Z_{-k}^{-j-1}=\omega_{-k}^{-j-1}\right) \times \\
& \quad \times \mathbb{P}\left(Z_{-j-1}=c \mid X_{-j-1}^{-1}=\omega_{-j-1}^{-1}, Z_{-k}^{-j-2}=\omega_{-k}^{-j-2}\right)
\end{aligned}
$$

We observe that the parcel corresponding to $c=\omega_{-j-1}$ of (4.13) is null. When $c=\omega^{\prime}{ }_{-j-1}$, with $\omega^{\prime}{ }_{-j-1} \neq \omega_{-j-1}$, the corresponding parcel of (4.13) can be bounded from above by $2 \beta_{j, X} \varepsilon$. From this, (4.12) and (4.9) we conclude the proof of Theorem 3.1.

\subsection{Proof of Theorem 3.2}

The proof of our second main result is based on four lemmas. The first one is consequence of Lemma 3.4 of Galves and Leonardi (2008). 
Lemma 4.4. There exists a summable sequence $\left(\rho_{l, X}\right)_{l \in \mathbb{N}}$, satisfying

$$
\sum_{l \in \mathbb{N}} \rho_{l, X} \leq 2\left(1+\frac{\beta_{X}}{\alpha_{X}}\right)
$$

such that for any $i \geq 1$, any $k \geq i$, any $j \geq 1$ and any finite sequence $\omega_{1}^{j}$, the following inequality holds

$$
\sup _{x_{1}^{i}, \theta_{1}^{i} \in \mathcal{A}^{i}}\left|\mathbb{P}\left(Z_{k}^{k+j-1}=\omega_{1}^{j} \mid X_{1}^{i}=x_{1}^{i}, \xi_{1}^{i}=\theta_{1}^{i}\right)-\mu_{Z}\left(\omega_{1}^{j}\right)\right| \leq \frac{\sum_{l=0}^{j-1} \rho_{k-i+l, X}}{(1-\varepsilon)^{j}},
$$

where $\alpha_{X}$ and $\beta_{X}$ are the same quantities as those in Definition 2.1.

Proof. Since the processes $\mathbf{X}, \mathbf{Y}$ and $\boldsymbol{\xi}$ are independent, we have

$$
\begin{aligned}
\mid \mathbb{P} & \left(Z_{k}^{k+j-1}=\omega_{1}^{j} \mid X_{1}^{i}=x_{1}^{i}, \xi_{1}^{i}=\theta_{1}^{i}\right)-\mu_{Z}\left(\omega_{1}^{j}\right) \mid \\
& =\left|\sum_{x_{k}^{k+j-1}} \mathbb{P}\left(X_{k}^{k+j-1}=x_{k}^{k+j-1}, Z_{k}^{k+j-1}=\omega_{1}^{j} \mid X_{1}^{i}=x_{1}^{i}, \xi_{1}^{i}=\theta_{1}^{i}\right)-\mu_{Z}\left(\omega_{1}^{j}\right)\right| \\
& =\left|\sum_{x_{k}^{k+j-1}} p_{X}\left(x_{k}^{k+j-1} \mid x_{1}^{i}\right) \mathbb{P}\left(Z_{k}^{k+j-1}=\omega_{1}^{j} \mid X_{k}^{k+j-1}=x_{k}^{k+j-1}\right)-\mu_{Z}\left(\omega_{1}^{j}\right)\right|,
\end{aligned}
$$

for any $x_{1}^{i}, \theta_{1}^{i} \in \mathcal{A}_{1}^{i}$. The last two summations are over the set

$$
\mathcal{C}^{*}=\left\{x_{k}^{k+j-1} \in \mathcal{A}_{1}^{j} \mid\left\{X_{k}^{k+j-1}=x_{k}^{k+j-1}, Z_{k}^{k+j-1}=\omega_{1}^{j}\right\} \neq \emptyset\right\} .
$$

On the other hand, we can see that

$$
\mu_{Z}\left(\omega_{1}^{j}\right)=\sum_{x_{k}^{k+j-1} \in \mathcal{C}^{*}} \mathbb{P}\left(Z_{k}^{k+j-1}=\omega_{1}^{j} \mid X_{k}^{k+j-1}=x_{k}^{k+j-1}\right) \mathbb{P}\left(X_{k}^{k+j-1}=x_{k}^{k+j-1}\right) .
$$

Then, by using the last identity, (4.15) and the Lemma 3.4 of Galves and Leonardi (2008), we can write

$$
\left|\mathbb{P}\left(Z_{k}{ }^{k+j-1}=\omega_{1}^{j} \mid X_{1}^{i}=x_{1}^{i}, \xi_{1}^{i}=\theta_{1}^{i}\right)-\mu_{Z}\left(\omega_{1}^{j}\right)\right| \leq \frac{\sum_{l=0}^{j-1} \rho_{k-i+l, X}}{(1-\xi)^{j}},
$$

where the sequence $\left(\rho_{l, X}\right)_{l \in \mathbb{N}}$ satisfies (4.14).

The proof of next result is a consequence of Proposition 4 of Dedecker and Doukhan (2003). 
Lemma 4.5. For any finite sequence $\omega$ and any $t>0$, we have

$$
\mathbb{P}\left(\left|N_{n}(\omega)-(n-l(\omega)+1) \mu_{Z}(\omega)\right|>t\right) \leq e^{\frac{1}{e}} \exp \left[-\frac{-t^{2}(1-\varepsilon)^{l(\omega)}}{4 e[n-l(\omega)+1] l(\omega)\left(1+\frac{\beta_{X}}{\alpha_{X}}\right)}\right] .
$$

Also, for any $a \in \mathcal{A}$ and any $n>\frac{|\mathcal{A}|+1}{t q(\omega)}+l(\omega)$, we have

$\left.\mathbb{P}\left(\left|\hat{p}_{(} a\right| \omega\right)_{n}-p_{Z}(a \mid \omega) \mid>t\right) \leq 3 e^{\frac{1}{e}} \exp \left\{-(n-l(\omega)) \frac{\left[t-\frac{3}{(n-l(\omega)) \mu_{Z}(\omega)}\right]^{2} \mu_{Z}(\omega)^{2}(1-\varepsilon)^{l(\omega a)}}{64 e l(\omega a)\left(1+\frac{\beta_{X}}{\alpha_{X}}\right)}\right\}$.

Proof. For the model (3.1) we have, for any finite sequence $\omega_{1}^{j} \in \mathcal{A}_{1}^{j}$, that

$$
N_{n}\left(\omega_{1}^{j}\right)=\sum_{t=0}^{n-j} \prod_{i \in \mathcal{I}^{*}}\left[\mathbf{1}_{\left\{X_{t+i}=\omega_{i}\right\}} \mathbf{1}_{\left\{\xi_{t+i}=1\right\}}\right] \prod_{s \in \mathcal{S}^{*}}\left[\mathbf{1}_{\left\{X_{t+s}=\omega_{s}\right\}}+\mathbf{1}_{\left\{X_{t+s}=1\right\}} \mathbf{1}_{\left\{\xi_{t+s}=0\right\}}\right],
$$

where $\mathcal{I}^{*}=\left\{k: 1 \leq k \leq j, \omega_{k} \neq 0\right\}$ and $\mathcal{S}^{*}=\left\{l: 1 \leq l \leq j, \omega_{l}=0\right\}$. Define the process $\mathbf{U}$ by

$$
U_{t}=\prod_{i \in \mathcal{I}^{*}}\left[\mathbf{1}_{\left\{X_{t+i}=\omega_{i}\right\}} \mathbf{1}_{\left\{\xi_{t+i}=1\right\}}\right] \prod_{s \in \mathcal{S}^{*}}\left[\mathbf{1}_{\left\{X_{t+s}=\omega_{s}\right\}}+\mathbf{1}_{\left\{X_{t+s}=1\right\}} \mathbf{1}_{\left\{\xi_{s+t}=0\right\}}\right]-\mu_{Z}\left(\omega_{1}^{j}\right) .
$$

Denote by $\mathcal{M}_{i}$ the $\sigma$-algebra generated by $U_{0}, \ldots, U_{i}$. Note that $\mathbb{E}\left(U_{t}\right)=0$ and $\left\|U_{t}\right\|_{\frac{r}{2}} \leq 1$. Now, by applying Proposition 4 of Dedecker and Doukhan (2003), we obtain

$$
\left\|N_{n}\left(\omega_{1}^{j}\right)-(n-j+1) \mu_{Z}\left(\omega_{1}^{j}\right)\right\|_{r} \leq\left(2 r \sum_{t=0}^{n-j} \max _{t \leq l \leq n-j}\left\|U_{t} \sum_{k=t}^{l} \mathbb{E}\left(U_{k} \mid \mathcal{M}_{t}\right)\right\|_{r / 2}\right)^{1 / 2},
$$

It follows by definition of $\|\cdot\|_{\infty}$ that

$$
\left\|\mathbb{E}\left(U_{k} \mid \mathcal{M}_{t}\right)\right\|_{\infty}=\sup _{x_{1}^{t+j}, \theta_{1}^{t+j}}\left|\mathbb{P}\left(Z_{k+j}^{k+1}=\omega_{j}^{1} \mid X_{1}^{t+j}=x_{1}^{t+j}, \xi_{1}^{t+j}=\theta_{1}^{t+j}\right)-\mu_{Z}\left(\omega_{1}^{j}\right)\right|
$$

where $x_{1}^{t+j}, \theta_{1}^{t+j} \in \mathcal{A}_{1}^{t+j}$. Plugging (4.18) into (4.17) and applying Lemma 4.4, we have

$$
\left\|N_{n}\left(\omega_{1}^{j}\right)-(n-j+1) p_{Z}\left(\omega_{1}^{j}\right)\right\|_{r} \leq\left[\frac{4 r}{(1-\varepsilon)^{j}}(n-j+1) j\left(1+\frac{\beta_{X}}{\alpha_{X}}\right)\right]^{1 / 2} .
$$

Now, let

$$
B=\frac{4}{(1-\varepsilon)^{j}}(n-j+1) j\left(1+\frac{\beta_{X}}{\alpha_{X}}\right) .
$$

Then, as in Dedecker e Prieur (2005) we obtain for any $t>0$ that

$$
\mathbb{P}\left(\left|N_{n}\left(\omega_{1}^{j}\right)-(n-j+1) \mu_{Z}\left(\omega_{1}^{j}\right)\right|>t\right) \leq \min \left(1,\left[\frac{r B}{t^{2}}\right]^{\frac{r}{2}}\right) .
$$


Now, following Galves and Leonard (2008), one can infer that

$$
\min \left(1,\left[\frac{r B}{t^{2}}\right]^{\frac{r}{2}}\right) \leq \exp \left\{-\frac{t^{2}}{e B}+e^{-1}\right\}
$$

Thus, by plugging (4.21) into (4.20) the first assertion of the lemma follows. In order to prove the second one, we note that

$$
\left|p_{Z}(a \mid \omega)-\frac{(n-l(\omega)) \mu_{Z}(\omega a)+1}{(n-l(\omega)) \mu_{Z}(\omega)+|\mathcal{A}|}\right| \leq \frac{|\mathcal{A}|+1}{(n-l(\omega)) \mu_{Z}(\omega)} .
$$

Thus, we can write

$$
\begin{aligned}
& \mathbb{P}\left(\left|p_{Z}(a \mid \omega)-\hat{p}_{Z}(a \mid \omega)_{n}\right|>t\right) \\
& \quad \leq \mathbb{P}\left(\left|\hat{p}_{Z}(a \mid \omega)_{n}-\frac{(n-l(\omega)) \mu_{Z}(\omega a)+1}{(n-l(\omega)) \mu_{Z}(\omega)+|\mathcal{A}|}\right|>t-\frac{|\mathcal{A}|+1}{(n-l(\omega)) \mu_{Z}(\omega)}\right),
\end{aligned}
$$

for any $n \geq \frac{|\mathcal{A}|+1}{t q(\omega)}+l(\omega)$. Making $t^{\prime}=t-\frac{|\mathcal{A}|+1}{(n-l(\omega)) \mu_{Z}(\omega)}$, we can see that

$$
\begin{aligned}
& \mathbb{P}\left(\left|\hat{p}_{Z}(a \mid \omega)_{n}-\frac{(n-l(\omega)) \mu_{Z}(\omega a)+1}{(n-l(\omega)) \mu_{Z}(\omega)+|\mathcal{A}|}\right|>t^{\prime}\right) \\
& \quad \leq \mathbb{P}\left(\left|N_{n}(\omega a)-(n-l(\omega)) \mu_{Z}(\omega a)\right|>\frac{t^{\prime}}{2}\left[(n-l(\omega)) \mu_{Z}(\omega)+|\mathcal{A}|\right]\right) \\
& \quad+\sum_{b \in \mathcal{A}} \mathbb{P}\left(\left|N_{n}(\omega b)-(n-l(\omega)) \mu_{Z}(\omega b)\right|>\frac{t^{\prime}}{2|\mathcal{A}|}\left[(n-l(\omega)) \mu_{Z}(\omega)+|\mathcal{A}|\right]\right) .
\end{aligned}
$$

Therefore, by applying the first assertion of this lemma in the second expression of last inequality we conclude the proof of this lemma.

Lemma 4.6. For any $\delta>2\left[1+\frac{4 \beta_{X}}{\min \left(\alpha_{X} \beta_{X}^{*}, 1\right)}\right] \varepsilon$, any $\omega \in \mathcal{T}_{X}, u \omega \in \hat{\mathcal{T}}_{n}^{\delta, d}$ and

$$
n>\frac{6}{\left\{\delta-2 \varepsilon\left[1+\frac{4 \beta_{X}}{\min \left(1,(1+\varepsilon) \alpha_{X} \beta_{X}^{*}\right)}\right]\right\} \alpha_{X}^{d}(1-\varepsilon)^{d}}+d
$$

we have

$$
\mathbb{P}\left(\Delta_{n}(u \omega)>\delta\right) \leq 12 e^{\frac{1}{e}} \exp \left\{-(n-d) \frac{\left[\frac{\delta}{2}-\bar{k}\right]^{2} \alpha_{X}^{2 d}(1-\varepsilon)^{3 d+1}}{64 e(d+1)\left(1+\frac{\beta_{X}}{\alpha_{X}}\right)}\right\}
$$

where

$$
\bar{k}=\varepsilon\left[1+\frac{4 \beta}{\min \left(1,(1+\varepsilon) \alpha_{X} \beta_{X}^{*}\right)}\right]+\frac{3}{(n-d) \alpha_{X}^{d}(1-\varepsilon)^{d}} .
$$


Proof. By the suffix property (item (2) of the Definition 2.2) if $\omega \in \mathcal{T}_{X}$ then

$$
p_{X}(a \mid u \omega)=p_{X}(a \mid \operatorname{suf}(u \omega)),
$$

for any finite sequence $u$ and any symbol $a \in \mathcal{A}$. Thus, from (4.24), by triangle inequality, we have

$$
\begin{aligned}
& \left|\hat{p}_{Z}(a \mid u \omega)_{n}-\hat{p}_{Z}\left(a \mid \operatorname{suf}(u \omega)_{n}\right)\right| \leq\left|\hat{p}_{Z}(a \mid u \omega)_{n}-p_{Z}(a \mid u \omega)\right|+\left|p_{X}(a \mid u \omega)-p_{Z}(a \mid u \omega)\right| \\
& \quad+\left|p_{X}(a \mid \operatorname{suf}(u \omega))-p_{Z}(a \mid \operatorname{suf}(u \omega))\right|+\left|p_{Z}(a \mid \operatorname{suf}(u \omega))-\hat{p}_{Z}(a \mid \operatorname{suf}(u \omega))_{n}\right| .
\end{aligned}
$$

Then, considering Theorem 3.1, the following inequality holds

$$
\begin{aligned}
& \mathbb{P}\left(\max _{a \in \mathcal{A}}\left|\hat{p}_{Z}(a \mid u \omega)_{n}-\hat{p}_{Z}(a \mid \operatorname{suf}(u \omega))_{n}\right|>\delta\right) \\
& \quad \leq \sum_{a \in \mathcal{A}}\left[\mathbb{P}\left(\left|\hat{p}_{Z}(a \mid u \omega)_{n}-p_{Z}(a \mid \operatorname{suf}(u \omega))\right|>\frac{\delta}{2}-\varepsilon\left[1+\frac{4 \beta_{X}}{\min \left(1,(1+\varepsilon) \alpha_{X} \beta_{X}^{*}\right)}\right]\right)\right. \\
& \left.\quad+\mathbb{P}\left(\left|p_{Z}(a \mid \operatorname{suf}(u \omega))-\hat{p}_{Z}(a \mid \operatorname{suf}(u \omega))_{n}\right|>\frac{\delta}{2}-\varepsilon\left[1+\frac{4 \beta_{X}}{\min \left(1,(1+\varepsilon) \alpha_{X} \beta_{X}^{*}\right)}\right]\right)\right] .
\end{aligned}
$$

Therefore, by using Lemmas 4.2 and 4.5 to bound from above the second expression of last inequality, the lemma follows.

Lemma 4.7. There exists $d$ such that, for any

$$
\delta<D_{d}-2 \varepsilon\left[1+\frac{4 \beta_{X}}{\min \left(1,(1+\varepsilon) \alpha_{X} \beta_{X}^{*}\right)}\right]
$$

any $\omega \in \hat{\mathcal{T}}_{n}^{\delta, d}$, with $l(\omega)<K, \omega \notin \mathcal{T}_{X}$ and any

$$
n>\frac{6}{\left\{D_{d}-\delta-2 \varepsilon\left[1+\frac{4 \beta_{X}}{\min \left(1,(1+\varepsilon) \alpha_{X} \beta_{X}^{*}\right)}\right]\right\} \alpha_{X}^{d}(1-\varepsilon)^{d}}+d,
$$

we have

$$
\mathbb{P}\left(\bigcap_{\left.u \omega \in \mathcal{T}_{X}\right|_{d}}\left\{\Delta_{n}(u \omega) \leq \delta\right\}\right) \leq 6 e^{\frac{1}{e}} \exp \left[-(n-d) \frac{\left[\frac{D_{d}-\delta}{2}-\bar{k}\right]^{2} \alpha_{X}^{2 d}(1-\varepsilon)^{3 d+1}}{64 e(d+1)\left(1+\frac{\beta_{X}}{\alpha_{X}}\right)}\right],
$$

where $\bar{k}$ is as in (4.23).

Proof. Similarly to Collet, Galves and Leonardi (2008), we take

$$
d=\max _{u \notin \mathcal{T}_{X}, l(u)<K} \min \left\{k: \text { existe } \omega \in C_{k} \operatorname{com} \omega \succ u\right\} .
$$


Then, from the definitions of $C_{d}$ and $d$, there exists $\left.\overline{u \omega} \in \mathcal{T}_{X}\right|_{d}$, such that $p_{X}(a \mid \bar{\omega} \omega) \neq$ $p_{X}(a \mid \operatorname{suf}(u \bar{\omega}))$ for some $a \in \mathcal{A}$. Now, we can see that

$$
\begin{aligned}
& \left|\hat{p}_{Z}(a \mid \overline{u \omega})_{n}-\hat{p}_{Z}(a \mid \operatorname{suf}(\overline{u \omega}))_{n}\right| \geq\left|p_{X}(a \mid \overline{u \omega})-p_{X}(a \mid \operatorname{suf}(u \bar{\omega}))\right| \\
& \quad-\left|\hat{p}_{Z}(a \mid \operatorname{suf}(\overline{u \omega}))_{n}-p_{Z}(a \mid \operatorname{suf}(\bar{u} \omega))\right|-\left|p_{Z}(a \mid \operatorname{suf}(\bar{u} \omega))-p_{X}(a \mid \operatorname{suf}(\bar{u} \omega))\right| \\
& \quad-\left|p_{Z}(a \mid u \bar{\omega})-p_{X}(a \mid \overline{u \omega})\right|-\left|\hat{p}_{Z}(a \mid \bar{u})_{n}-p_{Z}(a \mid u \bar{\omega})\right|
\end{aligned}
$$

for all $a \in \mathcal{A}$. Thus, if $\overline{u \omega} \in C_{d}$, then

$$
\max _{a \in \mathcal{A}}\left|p_{X}(a \mid \overline{u \omega})-p_{X}(a \mid \operatorname{suf}(\bar{u} \omega))\right| \geq D_{d}
$$

From Theorem 3.1, we can write

$$
\begin{aligned}
\Delta_{n}(u \bar{\omega}) & \geq D_{d}-2 \varepsilon\left[1+\frac{4 \beta_{X}}{\min \left(1,(1+\varepsilon) \alpha_{X} \beta_{X}^{*}\right)}\right] \\
& -\max _{a \in \mathcal{A}}\left|\hat{p}_{Z}(a \mid u \bar{\omega})_{n}-p_{Z}(a \mid \overline{u \omega})\right|-\max _{a \in \mathcal{A}}\left|\hat{p}_{Z}(a \mid \operatorname{suf}(\overline{u \omega}))_{n}-p_{Z}(a \mid \operatorname{suf}(\overline{u \omega}))\right|
\end{aligned}
$$

From the last inequality and Lemma 4.5 we have

$$
\mathbb{P}\left(\bigcap_{\left.u \omega \in \mathcal{T}_{X}\right|_{d}}\left\{\Delta_{n}(u \omega) \leq \delta\right\}\right) \leq 6 e^{\frac{1}{e}} \exp \left[-(n-d) \frac{c^{2} \alpha_{X}^{2 d}(1-\varepsilon)^{3 d+1}}{64 e(d+1)\left(1+\frac{\beta_{X}}{\alpha_{X}}\right)}\right],
$$

where $c=\frac{D_{d}-\delta}{2}-\varepsilon\left[1+\frac{4 \beta_{X}}{\min \left(1,(1+\varepsilon) \alpha_{X} \beta_{X}^{*}\right)}\right]-\frac{3}{(n-d) \alpha_{X}^{d}(1-\varepsilon)^{d}}$. This concludes the proof of this lemma.

Proof of Theorem 3.2. Following Bühlmann and Wyner (1999), we define

$$
O_{n, \delta}^{K, d}=\bigcup_{\substack{\omega \in \mathcal{T}_{X} \\ l(\omega)<K}} \bigcup_{u \omega \in \hat{\mathcal{T}}_{n}^{\delta, d}}\left\{\Delta_{n}(u \omega)>\delta\right\}
$$

and

$$
U_{n, \delta}^{K, d}=\bigcup_{\substack{\omega \in \hat{\mathcal{T}}_{n}^{\delta, d} \\ l(\omega)<K}} \bigcap_{\left.u \omega \in \mathcal{T}_{X}\right|_{d}}\left\{\Delta_{n}(u \omega) \leq \delta\right\}
$$

Then, if $d<n$, one can see that

$$
\left\{\left.\hat{\mathcal{T}}_{n}^{\delta, d}\right|_{K} \neq\left.\mathcal{T}_{X}\right|_{K}\right\} \subseteq O_{n, \delta}^{K, d} \cup U_{n, \delta}^{K, d}
$$

Thus,

$$
\mathbb{P}\left(\left.\hat{\mathcal{T}}_{n}^{\delta, d}\right|_{K} \neq\left.\mathcal{T}_{X}\right|_{K}\right) \leq \sum_{\substack{\omega \in \mathcal{T}_{X} \\ l(\omega)<K}} \sum_{u \omega \in \hat{\mathcal{T}}_{n}^{\delta, d}} \mathbb{P}\left(\Delta_{n}(u \omega)>\delta\right)+\sum_{\substack{\omega \in \hat{\mathcal{T}}_{1}^{\delta, d} \\ l(\omega)<K}} \mathbb{P}\left(\bigcap_{\left.u \omega \in \mathcal{T}_{X}\right|_{d}}\left\{\Delta_{n}(u \omega) \leq \delta\right\}\right)
$$


Therefore, from Definition 2.4, Lemmas 4.6 and 4.7, we have

$$
\mathbb{P}\left(\left.\hat{\mathcal{T}}_{n}^{\delta, d}\right|_{K} \neq\left.\mathcal{T}_{X}\right|_{K}\right) \leq 2^{d} 12 e^{\frac{1}{e}} \exp \left[-(n-d) \frac{\left[\min \left(D_{d}-\delta, \delta\right)-2 \bar{k}\right]^{2} \alpha_{X}^{2 d}(1-\varepsilon)^{3 d+1}}{256 e(d+1)\left(1+\frac{\beta_{X}}{\alpha_{X}}\right)}\right]
$$

where $\bar{k}$ is as in (4.23). It completes the proof of Theorem 3.2.

Proof of Corollary 3.3. By the Theorem 3.2, we have

$$
\sum_{n} \mathbb{P}\left(\left.\hat{\mathcal{T}}_{n}^{\delta, d}\right|_{K} \neq\left.\mathcal{T}_{X}\right|_{K}\right) \leq \sum_{n} c_{2} e^{\left[-(n-d) c_{3}\right]}<\infty
$$

for suitable choices of $d$ and $\delta$. Thus, by the Borel-Cantelli Lemma, we obtain

$$
\mathbb{P}\left(\left[\left.\hat{\mathcal{T}}_{n}^{\delta, d}\right|_{K} \neq\left.\mathcal{T}_{X}\right|_{K} \text { i.o. }\right]\right)=0 .
$$

\subsection{Proof of Theorem 3.4}

The proof of Theorem 3.4 is based on three preparatory lemmas.

Lemma 4.8. For any $\varepsilon \in(0,1)$, any $k>j \geq 0$, any $\omega_{-\infty}^{-a} \in \mathcal{A}_{-\infty}^{-1}, \omega_{0} \in \mathcal{A}$ and any $a, b \in \mathcal{A}$, we have

$\left|\mathbb{P}\left(X_{0}=\omega_{0} \mid X_{-j}^{-1}=\omega_{-j}^{-1}, X_{-j-1}=a, Z_{-j-1}=b, Z_{-k}^{-j-2}=\omega_{-k}^{-j-2}\right)-p_{X}\left(\omega_{0} \mid \omega_{-\infty}^{-1}\right)\right| \leq \beta_{j, X}$.

Proof. For any $j \geq 0$, considering the independence of the processes $\boldsymbol{\xi}, \mathbf{Y}$ and $\mathbf{X}$, we have

$$
\begin{gathered}
\mathbb{P}\left(X_{0}=\omega_{0} \mid X_{-j}^{-1}=\omega_{-j}^{-1}, X_{-j-1}=a, Z_{-j-1}=b, Z_{-k}^{-j-2}=\omega_{-k}^{-j-2}\right) \\
=\frac{\sum_{-k}^{-j-2} p_{X}\left(u_{-k}^{-j-2} a \omega_{-j}^{-1} \omega_{0}\right) \mathbb{P}\left(Z_{-k}^{-j-1}=\omega_{-k}^{-j-2} b \mid X_{-k}^{-j-1}=u_{-k}^{-j-2} a\right)}{\sum_{u_{-k}^{-j-2}} p_{X}\left(u_{-k}^{-j-2} a \omega_{-j}^{-1}\right) \mathbb{P}\left(Z_{-k}^{-j-1}=\omega_{-k}^{-j-2} b \mid X_{-k}^{-j-1}=u_{-k}^{-j-2} a\right)} .
\end{gathered}
$$

Since

$$
p_{X}\left(\omega_{0} \mid \omega_{-\infty}^{-1}\right)-\beta_{j, X} \leq p_{X}\left(\omega_{0} \mid u_{-k}^{-j-2} a \omega_{-j}^{-1}\right) \leq p_{X}\left(\omega_{0} \mid \omega_{-\infty}^{-1}\right)+\beta_{j, X},
$$

the assertion of this lemma follows directly.

Lemma 4.9. For any $\varepsilon \in(0,1)$, any $k \geq 0$ and any $\omega_{-k}^{0}$, we have

$$
\begin{gathered}
p_{Z}\left(\omega_{0} \mid \omega_{-k}^{-1}\right) \geq \alpha_{\min }, \\
\mathbb{P}\left(X_{0}=\omega_{0} \mid Z_{-k}^{-1}=\omega_{-k}^{-1}\right) \geq \alpha_{X},
\end{gathered}
$$




$$
\mathbb{P}\left(Y_{0}=\omega_{0} \mid Z_{-k}^{-1}=\omega_{-k}^{-1}\right) \geq \alpha_{Y},
$$

where $\alpha_{\min }=\min \left\{\alpha_{X}, \alpha_{Y}\right\}$. Moreover, for any $0 \leq j \leq k$, we have

$$
\mathbb{P}\left(X_{-j-1}=\omega_{-j-1} \mid X_{-j}^{-1}=\omega_{-j}^{-1}, Z_{-k}^{-j-2}=\omega_{-k}^{-j-2}\right) \geq \alpha_{X} \beta_{X}^{*},
$$

where $\beta_{X}^{*}=\prod_{k=0}^{+\infty}\left(1-\beta_{k, X}\right)>0$.

Proof. It follows from (3.3) that

$$
p_{Z}\left(\omega_{0} \mid \omega_{-k}^{-1}\right)=(1-\varepsilon) \mathbb{P}\left(X_{0}=\omega_{0} \mid Z_{-k}^{-1}=\omega_{-k}^{-1}\right)+\varepsilon \mathbb{P}\left(Y_{0}=\omega_{0} \mid Z_{-k}^{-1}=\omega_{-k}^{-1}\right) .
$$

Thus the assertion (4.25) follows from (4.26), (4.27) and (4.29). Now, considering the independence of the processes $\boldsymbol{\xi}, \mathbf{X}$ and $\mathbf{Y}$, we have

$$
\begin{array}{r}
\mathbb{P}\left(X_{0}=\omega_{0} \mid Z_{-k}^{-1}=\omega_{-k}^{-1}\right) \\
=\lim _{l \rightarrow+\infty} \frac{\sum_{v_{-k}^{-1}} \sum_{-l}^{-1} p_{X}\left(\omega_{0} \mid \omega_{-\infty}^{-l-1} u_{-l}^{-1}\right) \mathbb{P}\left(X_{-l}^{-1}=u_{-l}^{-1} \mid X_{-\infty}^{-l-1}=\omega_{-\infty}^{-l-1}\right) p_{Y}\left(v_{-k}^{-1}\right) p_{\xi}}{\sum_{v_{-k}^{-1}} \sum_{u_{-l}^{-1}} \mathbb{P}\left(X_{-l}^{-1}=u_{-l}^{-1} \mid X_{-\infty}^{-l-1}=\omega_{-\infty}^{l-1}\right) p_{Y}\left(v_{-k}^{-1}\right) p_{\xi}},
\end{array}
$$

where

$$
p_{\xi}=\mathbb{P}\left(\bigcap_{i:-k \leq-i \leq-1, u_{-i} \neq v_{-i}, u_{-i}=\omega_{-i}} \xi_{-i}=1, \bigcap_{t:-k \leq-t \leq-1,} \bigcap_{u_{-t} \neq v_{-t}, u_{-t} \neq \omega_{-t}} \xi_{-t}=0\right),
$$

with the sequences $u_{-l}^{-1} \in \mathcal{A}_{-l}^{-1}, v_{-k}^{-1} \in \mathcal{A}_{-k}^{-1}$, satisfying

$$
\mathbb{P}\left(X_{-l}^{-1}=u_{-l}^{-1}, Y_{-k}^{-1}=v_{-k}^{-1}, Z_{-k}^{-1}=\omega_{-k}^{-1}\right) \neq 0 .
$$

From the non-nullness hypothesis of the process $\mathbf{X}$ and (4.30) we can write (4.26). Analogously, we can prove (4.27). Now, in order to prove (4.28), we note that

$$
\begin{aligned}
\mathbb{P} & \left(X_{-j-1}=\omega_{-j-1} \mid X_{-j}^{-1}=\omega_{-j}^{-1}, Z_{-k}^{-j-2}=\omega_{-k}^{-j-2}\right) \\
& =\frac{\sum \mathbb{P}\left(Z_{-k}^{-j-2}=\omega_{-k}^{-j-2} \mid X_{-k}^{-j-2}=x_{-k}^{-j-2}, Y_{-k}^{-j-2}=y_{-k}^{-j-2}\right) p_{X}\left(x_{-k}^{-j-2} \omega_{-j-1}^{-1}\right) p_{Y}\left(y_{-k}^{-j-2}\right)}{\sum \mathbb{P}\left(Z_{-k}^{-j-2}=\omega_{-k}^{-j-2} \mid X_{-k}^{-j-2}=x_{-k}^{-j-2}, Y_{-k}^{-j-2}=y_{-k}^{-j-2}\right) p_{X}\left(x_{-k}^{-j-2} \omega_{-j}^{-1}\right) p_{Y}\left(y_{-k}^{-j-2}\right)} .
\end{aligned}
$$

The last two summations are over the set

$$
\mathcal{S}^{*}=\left\{x_{-k}^{-j-2}, y_{-k}^{-j-2} \in \mathcal{A}_{-k}^{-j-2} \mid\left\{X_{-k}^{-j-2}=x_{-k}^{-j-2}, Y_{-k}^{-j-2}=y_{-k}^{-j-2}, Z_{-k}^{-j-2}=\omega_{-k}^{-j-2}\right\} \neq \emptyset\right\} .
$$

Therefore, by plugging (4.8) into (4.31), the assertion (4.28) follows. 
Lemma 4.10. For any $\varepsilon \in(0,1)$, any $k>j \geq 0$ and any $\omega_{-k}^{0}$, we have

$$
\mathbb{P}\left(X_{-j-1}=\omega_{-j-1}^{\prime} \mid X_{-j}^{-1}=\omega_{-j}^{-1}, Z_{-k}^{-j-1}=\omega_{-k}^{-j-1}\right) \leq \frac{\varepsilon}{\alpha \beta_{\min }^{*}},
$$

where $\omega_{-j-1}^{\prime} \neq \omega_{-j-1}$ and $\alpha \beta_{\min }^{*}=\min \left\{\alpha_{X} \beta_{X}^{*}, \alpha_{Y}\right\}$.

Proof. Analogously to the proof of Lemma 4.3, we can show that

$$
\begin{aligned}
& \mathbb{P}\left(X_{-j-1}=\omega_{-j-1}^{\prime} \mid X_{-j}^{-1}=\omega_{-j}^{-1}, Z_{-k}^{-j-1}=\omega_{-k}^{-j-1}\right) \\
& \quad \leq \frac{\varepsilon}{\mathbb{P}\left(Z_{-j-1}=\omega_{-j-1} \mid X_{-j}^{-1}=\omega_{-j}^{-1}, Z_{-k}^{-j-2}=\omega_{-k}^{-j-2}\right)} .
\end{aligned}
$$

Now, from the independence between the processes $\mathbf{X}$ and $\mathbf{Y}$, the inequality (4.27), it follows that

$$
\mathbb{P}\left(Y_{-j-1}=\omega_{-j-1} \mid X_{-j}^{-1}=\omega_{-j}^{-1}, Z_{-k}^{-j-2}=\omega_{-k}^{-j-2}\right) \geq \alpha_{Y} .
$$

From (4.33), (4.28) and (4.32), the lemma is proved.

Proof of Theorem 3.4. First, for any $a \in \mathcal{A}$ and any $\omega_{-k}^{-1} \in \mathcal{A}_{-k}^{-1}$, we have

$$
\left|p_{Z}\left(a \mid \omega_{-k}^{-1}\right)-\mathbb{P}\left(X_{0}=a \mid Z_{-k}^{-1}=\omega_{-k}^{-1}\right)\right| \leq 2 \varepsilon .
$$

Proceeding analogously to the proof of Theorem 3.1, taking account Lemmas 4.8 and 4.10, one can show that

$$
\mathbb{P}\left(X_{0}=a \mid Z_{-k}^{-1}=\omega_{-k}^{-1}\right)-\mathbb{P}\left(X_{0}=a \mid X_{-k}^{-1}=\omega_{-k}^{-1}\right) \leq \sum_{j=0}^{k-1}\left[(N-1) \frac{2 \varepsilon \beta_{j, X}}{\alpha \beta_{\min }^{*}}+(N-1) 2 \varepsilon \beta_{j, X}\right] .
$$

Therefore, considering the last inequality and (4.34), we have

$$
\left|\mathbb{P}\left(X_{0}=a \mid Z_{-k}^{-1}=\omega_{-k}^{-1}\right)-\mathbb{P}\left(X_{0}=a \mid X_{-k}^{-1}=\omega_{-k}^{-1}\right)\right| \leq 2 \varepsilon+(N-1)\left[\frac{2 \varepsilon \beta_{X}}{\alpha \beta_{\min }^{*}}+2 \varepsilon \beta_{X}\right]
$$

and the theorem is proved.

\subsection{Proof of Theorem 3.5}

Lemma 4.11. For any $i \geq 1$, any $k \geq i$, any $j \geq 1$ and any finite sequence $\omega_{1}^{j} \in \mathcal{A}_{1}^{j}$, the following inequality holds

$$
\sup _{x_{1}^{i}, y_{1}^{i} \in \mathcal{A}^{i} ; \theta_{1}^{i} \in\{0,1\}^{i}}\left|\mathbb{P}\left(Z_{k}^{k+j-1}=\omega_{1}^{j} \mid X_{1}^{i}=x_{1}^{i}, Y_{1}^{i}=y_{1}^{i}, \xi_{1}^{i}=\theta_{1}^{i}\right)-p_{Z}\left(\omega_{1}^{j}\right)\right| \leq \frac{2 \rho_{i j k, \max }}{(1-\varepsilon)^{j}},
$$

where

$$
\rho_{i j k, \max }=\max \left\{\sum_{l=0}^{j-1} \rho_{k-i+l, X}, \sum_{s=0}^{j-1} \rho_{k-i+s, Y}\right\}
$$


Proof. From the independence of processes $\mathbf{X}, \mathbf{Y}$ and $\boldsymbol{\xi}$, for any $x_{1}^{i}, y_{1}^{i} \in \mathcal{A}^{i}$ and any $\theta_{1}^{i} \in\{0,1\}^{i}$, we have

$$
\begin{aligned}
\mid \mathbb{P}( & \left.Z_{k}{ }^{k+j-1}=\omega_{1}^{j} \mid X_{1}^{i}=x_{1}^{i}, Y_{1}^{i}=y_{1}^{i}, \xi_{1}^{i}=\theta_{1}^{i}\right)-p_{Z}\left(\omega_{1}^{j}\right) \mid \\
= & \mid \sum \mathbb{P}\left(X_{k}^{k+j-1}=x_{k}^{k+j-1}, Y_{k}^{k+j-1}=y_{k}^{k+j-1}, Z_{k}^{k+j-1}=\omega_{1}^{j} \mid X_{1}^{i}=x_{1}^{i}, Y_{1}^{i}=y_{1}^{i}, \xi_{1}^{i}=\theta_{1}^{i}\right) \\
& -p_{Z}\left(\omega_{1}^{j}\right) \mid \\
& \quad \times\left|\mathbb{P}\left(X_{k}^{k+j-1}=x_{k}^{k+j-1}, Y_{k}^{k+j-1}=y_{k}^{k+j-1} \mid X_{1}^{i}=x_{1}^{i}, Y_{1}^{i}=y_{1}^{i}\right)-p_{X}\left(x_{k}^{k+j-1}\right) p_{Y}\left(y_{k}^{k+j-1}\right)\right| \\
\leq & \sum \mathbb{P}\left(Z_{k}^{k+j-1}=\omega_{1}^{j} \mid X_{k}^{k+j-1}=x_{k}^{k+j-1}, Y_{k}^{k+j-1}=y_{k}^{k+j-1}\right) \\
& \quad \times\left[\mid \mathbb{P}\left(X_{k}^{k+j-1}=x_{k}^{k+j-1}, Y_{k}^{k+j-1}=y_{k}^{k+j-1} \mid X_{1}^{i}=x_{1}^{i}, Y_{1}^{i}=y_{1}^{i}\right)\right. \\
& -p_{Y}\left(y_{k}^{k+j-1} \mid y_{1}^{i}\right) p_{X}\left(x_{k}^{k+j-1}\right) \mid \\
& \left.+\left|p_{Y}\left(y_{k}^{k+j-1} \mid y_{1}^{i}\right) p_{X}\left(x_{k}^{k+j-1}\right)-p_{X}\left(x_{k}^{k+j-1}\right) p_{Y}\left(y_{k}^{k+j-1}\right)\right|\right] \\
\leq & \frac{2 \rho_{i j k, \max }}{(1-\xi)^{j}}
\end{aligned}
$$

where the summations are over $x_{k}^{k+j-1}, y_{k}^{k+j-1} \in \mathcal{A}_{1}^{j}$. Since the $\mathbf{X}$ and $\mathbf{Y}$ satisfy the assumptions of non-nullness and summability of the continuity rate the last inequality is a consequence the Lemma 3.4 of Galves and Leonardi (2008).

We observe that from this point on the technique used in the proof of the Theorem 3.5 will be essentially the same employed in the proof of Theorem 3.2.

Lemma 4.12. For any finite sequence $\omega$ and any $t>0$, we have

$$
\mathbb{P}\left(\left|N_{n}(\omega)-(n-l(\omega)+1) p_{Z}(\omega)\right|>t\right) \leq e^{\frac{1}{e}} \exp \left[-\frac{-t^{2}(1-\varepsilon)^{l(\omega)}}{4 e[n-l(\omega)+1] l(\omega) \beta_{\alpha, \max }}\right],
$$

where $\beta_{\alpha, \max }=\max \left\{\left(1+\frac{\beta_{X}}{\alpha_{X}}\right),\left(1+\frac{\beta_{Y}}{\alpha_{Y}}\right)\right\}$. Moreover, for any $a \in \mathcal{A}$ and any $n>$ $\frac{N+1}{t q(\omega)}+l(\omega)$, we have

$$
\begin{aligned}
& \mathbb{P}\left(\left|\hat{p}_{Z_{n}}(a \mid \omega)-p_{Z}(a \mid \omega)\right|>t\right) \\
& \quad \leq(N+1) e^{\frac{1}{e}} \exp \left\{-\left[t-\frac{N+1}{(n-l(\omega)) p_{Z}(\omega)}\right]^{2} \frac{\left[p_{Z}(\omega)\right]^{2}(n-l(\omega))(1-\varepsilon)^{l(\omega a)}}{32 N^{2} e l(\omega a) \beta_{\alpha, \max }}\right\}
\end{aligned}
$$

Proof. Considering (2.4) and (3.3) we have, for any finite sequence $\omega_{1}^{j} \in \mathcal{A}^{j}$,

$$
N_{n}\left(\omega_{1}^{j}\right)=\sum_{t=0}^{n-j} \prod_{1 \leq i \leq j}\left[\mathbf{1}_{\left\{X_{t+i}=\omega_{i}\right\}} \mathbf{1}_{\left\{\xi_{t+i}=1\right\}}+\mathbf{1}_{\left\{Y_{t+i}=\omega_{i}\right\}} \mathbf{1}_{\left\{\xi_{t+i}=0\right\}}\right] .
$$


We define the process $\mathbf{U}$ by

$$
U_{t}=\prod_{1 \leq i \leq j}\left[\mathbf{1}_{\left\{X_{t+i}=\omega_{i}\right\}} \mathbf{1}_{\left\{\xi_{t+i}=1\right\}}+\mathbf{1}_{\left\{Y_{t+i}=\omega_{i}\right\}} \mathbf{1}_{\left\{\xi_{t+i}=0\right\}}\right]-p_{Z}\left(\omega_{1}^{j}\right)
$$

and we denote by $\mathcal{M}_{i}$ the $\sigma$-algebra generated by $U_{0}, \ldots, U_{i}$. Applying Proposition 4 of Dedecker and Doukhan (2003), we obtain

$$
\left\|N_{n}\left(\omega_{1}^{j}\right)-(n-j+1) p_{Z}\left(\omega_{1}^{j}\right)\right\|_{r} \leq\left(2 r \sum_{t=0}^{n-j}\left\|U_{t}\right\|_{r / 2} \sum_{k=t}^{l}\left\|\mathbb{E}\left(U_{k} \mid \mathcal{M}_{t}\right)\right\|_{\infty}\right)^{1 / 2},
$$

for any $r \geq 2$. We note that $\left\|U_{t}\right\|_{r / 2} \leq 1$. Moreover, for any $x_{1}^{t+j}, y_{1}^{t+j} \in \mathcal{A}_{1}^{t+j}$ and any $\theta_{1}^{t+j} \in\{0,1\}^{t+j}$, we have

$$
\left\|\mathbb{E}\left(U_{k} \mid \mathcal{M}_{t}\right)\right\|_{\infty}=\sup _{x_{1}^{t+j}, y_{1}^{t+j}, \theta_{1}^{t+j}}\left|\mathbb{P}\left(Z_{k+j}^{k+1}=\omega_{j}^{1} \mid X_{1}^{t+j}=x_{1}^{t+j}, X_{1}^{t+j}=y_{1}^{t+j}, \xi_{1}^{t+j}=\theta_{1}^{t+j}\right)-p_{Z}\left(\omega_{1}^{j}\right)\right| .
$$

Thus, by Lemmas 4.11 and 4.4 we have the following inequality

$$
\left\|N_{n}(\omega)-(n-j+1) p_{Z}(\omega)\right\|_{r} \leq\left[\frac{8 r}{(1-\varepsilon)^{l(\omega)}}(n-l(\omega)+1) l(\omega) \beta_{\alpha, \max }\right]^{1 / 2},
$$

where $\beta_{\alpha, \max }=\max \left\{\left(1+\frac{\beta_{X}}{\alpha_{X}}\right),\left(1+\frac{\beta_{Y}}{\alpha_{Y}}\right)\right\}$. Thus, as in proof of Lemma 4.5, we can write

$$
\mathbb{P}\left(\left|N_{n}(\omega)-(n-l(\omega)+1) p_{Z}(\omega)\right|>t\right) \leq e^{\frac{1}{e}} \exp \left\{-\frac{t^{2}(1-\varepsilon)^{l(\omega)}}{8 e(n-l(\omega)+1) l(\omega) \beta_{\alpha, \max }}\right\} .
$$

Now, one can see that the following inequality holds

$$
\begin{aligned}
& \mathbb{P}\left(\left|\frac{N_{n}(\omega a)+1}{N_{n}(\omega .)+|\mathcal{A}|}-\frac{(n-l(\omega)) p_{Z}(\omega a)+1}{(n-l(\omega)) p_{Z}(\omega)+|\mathcal{A}|}\right|>t^{\prime}\right) \\
& \quad \leq \mathbb{P}\left(\left|N_{n}(\omega a)-(n-l(\omega)) p_{Z}(\omega a)\right|>\frac{t^{\prime}}{2}\left[(n-l(\omega)) p_{Z}(\omega)+|\mathcal{A}|\right]\right) \\
& \quad+\sum_{b \in \mathcal{A}} \mathbb{P}\left(\left|N_{n}(\omega b)-(n-l(\omega)) p_{Z}(\omega b)\right|>\frac{t^{\prime}}{2|\mathcal{A}|}\left[(n-l(\omega)) p_{Z}(\omega)+|\mathcal{A}|\right]\right) .
\end{aligned}
$$

Therefore, applying (4.35) we can bound from above (4.36) by

$$
(N+1) e^{\frac{1}{e}} \exp \left\{-\left[t-\frac{N+1}{(n-l(\omega)) p_{Z}(\omega)}\right]^{2} \frac{\left[p_{Z}(\omega)\right]^{2}(n-l(\omega))(1-\varepsilon)^{l(\omega a)}}{32 N^{2} e(l(\omega a)) \beta_{\alpha, \max }}\right\} .
$$


Lemma 4.13. For any $\delta>4\left[1+\frac{2(N-1) \beta_{X}}{\min \left(1, \alpha \beta_{\text {min }}^{*}\right)}\right] \varepsilon$, any $\omega \in \mathcal{T}_{X}$, any $u \omega \in \hat{\mathcal{T}}_{n}^{\delta, d}$ and

$$
n>\frac{2(N+1)}{\left.\left\{\delta-4 \varepsilon\left[1+\frac{2(N-1) \beta_{X}}{\min \left(1, \alpha \beta_{\text {min }}^{*}\right.}\right)\right]\right\} \alpha_{\min }^{d}}+d
$$

we have

$$
\mathbb{P}\left(\Delta_{n}(u \omega)>\delta\right) \leq 2 N(N+1) e^{\frac{1}{e}} \exp \left\{-(n-d) \frac{\left[\frac{\delta}{2}-\bar{k}\right]^{2} \alpha_{\min }^{2 d}(1-\varepsilon)^{d+1}}{32 N^{2} e(d+1) \beta_{\alpha, \max }}\right\}
$$

where

$$
\bar{k}=2 \varepsilon\left[1+\frac{2(N-1) \beta_{X}}{\min \left(1, \alpha \beta_{\min }^{*}\right)}\right]+\frac{N+1}{(n-d) \alpha_{\min }^{d}} .
$$

Proof. Similarly to the proof of Lemma 4.6 we can show that

$$
\begin{aligned}
& \mathbb{P}\left(\Delta_{n}(u \omega)>\delta\right) \\
& \quad \leq \sum_{a \in \mathcal{A}}\left[\mathbb{P}\left(\left|\hat{p}_{Z}(a \mid u \omega)_{n}-p_{Z}(a \mid \operatorname{suf}(u \omega))\right|>\frac{\delta}{2}-2 \varepsilon\left[1+\frac{2(N-1) \beta_{X}}{\min \left(1, \alpha \beta_{\min }^{*}\right)}\right]\right)\right. \\
& \left.\quad+\mathbb{P}\left(\left|p_{Z}(a \mid \operatorname{suf}(u \omega))-\hat{p}_{Z}(a \mid \operatorname{suf}(u \omega))_{n}\right|>\frac{\delta}{2}-2 \varepsilon\left[1+\frac{2(N-1) \beta_{X}}{\min \left(1, \alpha \beta_{\min }^{*}\right)}\right]\right)\right] .
\end{aligned}
$$

Then, by using Lemma 4.12 we can bound from above the right side of the last inequality obtaining (4.37).

Lemma 4.14. There exists $d$ such that for any $\delta<D_{d}-4 \varepsilon\left[1+\frac{2(N-1) \beta_{X}}{\min \left(1, \alpha \beta_{\min }^{*}\right)}\right]$, any $\omega \in \hat{\mathcal{T}}_{n}^{\delta, d}$, with $l(\omega)<K, \omega \notin \mathcal{T}_{X}$, and any

$$
n>\frac{2(N+1)}{\left\{D_{d}-\delta-4 \varepsilon\left[1+\frac{2(N-1) \beta_{X}}{\min \left(1, \alpha \beta_{\min }^{*}\right)}\right]\right\} \alpha^{d}}+d
$$

we have

$$
\mathbb{P}\left(\bigcap_{\left.u \omega \in T\right|_{d}}\left\{\Delta_{n}(u \omega) \leq \delta\right\}\right) \leq 2(N+1) e^{\frac{1}{e}} \exp \left[-(n-d) \frac{\left[D_{d}-\delta-\bar{k}\right]^{2} \alpha_{\min }^{2 d}(1-\varepsilon)^{d}}{128 N^{2} e(d+1) \beta_{\alpha, \max }}\right]
$$

where $\bar{k}$ is as in Lemma 4.13 .

Proof. Take

$$
d=\max _{u \notin \mathcal{T}_{X}, l(u)<K} \min \left\{k: \text { there exists } \omega \in C_{k} \text { with } \omega \succ u\right\}
$$


As in proof of Lemma 4.7 we can show that

$$
\begin{aligned}
& \mathbb{P}\left(\Delta_{n}(\overline{u \omega}) \leq \delta\right) \\
& \quad \leq \mathbb{P}\left(\bigcap_{a \in \mathcal{A}}\left\{\left|\hat{p}_{Z}(a \mid \bar{\omega})_{n}-p_{Z}(a \mid \bar{\omega})\right| \geq \frac{D_{d}-\delta-4 \varepsilon\left[1+\frac{2(N-1) \beta_{X}}{\min \left(1, \alpha \beta_{\text {min }}^{*}\right)}\right]}{2}\right\}\right) \\
& \quad+\mathbb{P}\left(\bigcap_{a \in \mathcal{A}}\left\{\left|\hat{p}_{Z}(a \mid \overline{u \omega})_{n}-p_{Z}(a \mid \bar{\omega})\right| \geq \frac{D_{d}-\delta-4 \varepsilon\left[1+\frac{2(N-1) \beta_{X}}{\min \left(1, \alpha \beta_{\min }^{*}\right)}\right]}{2}\right\}\right) .
\end{aligned}
$$

Therefore, if $\delta<D_{d}-4 \varepsilon\left[1+\frac{2(N-1) \beta_{X}}{\min \left(1, \alpha \beta_{\min }^{*}\right)}\right]$ and

$$
n>\frac{2(N+1)}{\left\{D_{d}-\delta-4 \varepsilon\left[1+\frac{2(N-1) \beta_{X}}{\min \left(1, \alpha \beta_{\min }^{*}\right)}\right]\right\} \alpha^{d}}+d
$$

we can use Lemma 4.12 to conclude the proof of this lemma.

Proof of Theorem 3.5. By proceeding as in the proof of Theorem 3.2 we can show that

$$
\mathbb{P}\left(\left.\hat{\mathcal{T}}_{n}^{\delta, d}\right|_{K} \neq\left.\mathcal{T}_{X}\right|_{K}\right) \leq \sum_{\substack{\omega \in \mathcal{T}_{X} \\ l(\omega)<K}} \sum_{\substack{u \in \hat{\mathcal{T}}_{3 n}^{\delta, d}}} \mathbb{P}\left(\Delta_{n}(u \omega)>\delta\right)+\sum_{\substack{\omega \in \hat{\mathcal{T}}_{n}^{\delta, d} \\ l(\omega)<K}} \mathbb{P}\left(\bigcap_{\left.u \omega \in \mathcal{T}_{X}\right|_{d}}\left\{\Delta_{n}(u \omega) \leq \delta\right\}\right)
$$

if $d<n$. Therefore, by means of Lemmas 4.13 and 4.14 we obtain

$$
\mathbb{P}\left(\left.\hat{\mathcal{T}}_{n}^{\delta, d}\right|_{K} \neq\left.\mathcal{T}_{X}\right|_{K}\right) \leq c_{2} \exp \left\{-c_{3}(n-d)\right\}
$$

where $c_{2}=48 N^{d}(N+1) e^{\frac{1}{e}}$ and $c_{3}=\frac{\left[\min \left(D_{d}-\delta, \delta\right)-2 \bar{k}\right]^{2} \alpha^{2 d}}{128 N^{2} e(d+1) \beta_{\alpha, \max }}$.

Proof of Corollary 3.6. It follows from Theorem 3.5, First Borel-Cantelli Lemma and the fact that the quotas for the estimation error of the truncated context tree are summable at $n$ for appropriate choices of $d$ and $\delta$.

\section{Comparisons}

In this section we compare the results obtained in this paper with the corresponding ones presented in Collet, Galves and Leonardi (2008). For this purpose, let $\mathbf{X}$ and $\mathbf{Y}$ be independent processes taking values on the alphabet $\mathcal{A}=\{0,1\}$. Furthermore, we assume that these processes are non-null and have summable continuity rate with the same constants $\alpha_{X}$ and $\beta_{X}$.

To compare the bounds we are going to couple the processes using the same Bernoulli sequence $\boldsymbol{\xi}$ independent of the processes $\mathbf{X}$ and $\mathbf{Y}$, with $\mathbb{P}\left(\xi_{t}=1\right)=1-\varepsilon$, where $\varepsilon$ is fixed 
in $(0,1)$. Now, we define the stochastically perturbed chains $\mathbf{Z}_{\mathbf{1}}, \mathbf{Z}_{\mathbf{2}}$ and $\mathbf{Z}_{\mathbf{3}}$ by

$$
\begin{aligned}
& Z_{1, t}=X_{t}+\left(1-\xi_{t}\right)(\bmod 2), \\
& Z_{2, t}=X_{t} \cdot \xi_{t}, \\
& Z_{3, t}=\left\{\begin{array}{cl}
X_{t}, & \text { if } \xi_{t}=1, \\
Y_{t}, & \text { if } \xi_{t}=0,
\end{array}\right.
\end{aligned}
$$

where $t \in \mathbb{Z}$. The model (5.1) was proposed in Collet, Galves and Leonardi (2008). We assume that $\mathbf{Z}_{\mathbf{i}}$ is compatible with $q_{i}(\cdot \mid \cdot)$ the law of the process, for $i=1,2$ or 3 .

In the model (5.1), the process $\mathbf{Z}_{\mathbf{1}}$ will be different from the process $\mathbf{X}$ whenever $\xi_{t}=0$, which occurs with probability $\varepsilon$. The process $\mathbf{Z}_{\mathbf{2}}$, defined by (5.2) is equal to the process

$\mathbf{X}$ with high probability $1-\varepsilon$. The third contamination model, defined in (5.3), is such that at each instant of time the process $\mathbf{Z}_{\mathbf{3}}$ either is equal to the process $\mathbf{X}$, with high probability $1-\varepsilon$, or is equal to the process $\mathbf{Y}$, with small probability $\varepsilon$.

Theorem 1 of Collet, Galves and Leonardi (2008) states that

$$
k_{1}:=\varepsilon\left[1+\frac{4 \beta_{X}}{\min \left(1, \alpha_{X} \beta_{X}^{*}\right)}\right] \geq \sup _{a \in \mathcal{A}, \omega_{-k}^{-1} \in \mathcal{A}_{-k}^{-1}}\left|q_{1}\left(a \mid \omega_{-k}^{-1}\right)-p_{X}\left(a \mid \omega_{-k}^{-1}\right)\right|,
$$

where $\varepsilon \in(0,1), k \geq 0$ and $\beta_{X}^{*}=\prod_{k=0}^{+\infty}\left(1-\beta_{k, X}\right)<+\infty$. Our Theorem 3.1 says that

$$
k_{2}:=\varepsilon\left[1+\frac{4 \beta_{X}}{\min \left(1,(1+\varepsilon) \alpha_{X} \beta_{X}^{*}\right)}\right] \geq \sup _{a \in \mathcal{A}, \omega_{-k}^{-1} \in \mathcal{A}_{-k}^{-1}}\left|q_{2}\left(a \mid \omega_{-k}^{-1}\right)-p_{X}\left(a \mid \omega_{-k}^{-1}\right)\right| .
$$

Theorem 3.4 says that

$$
k_{3}:=\varepsilon\left[2+\frac{4 \beta_{X}}{\min \left(1, \alpha \beta_{\min }^{*}\right)}\right] \geq \sup _{a \in \mathcal{A}, \omega_{-k}^{-1} \in \mathcal{A}_{-k}^{-1}}\left|q_{3}\left(a \mid \omega_{-k}^{-1}\right)-p_{X}\left(a \mid \omega_{-k}^{-1}\right)\right|,
$$

where $\alpha \beta_{\min }^{*}=\min \left\{\alpha_{X} \beta_{X}^{*}, \alpha_{X}\right\}$. It is easy to see that

$$
k_{2} \leq k_{1} \leq k_{3} .
$$

We observe that the inequality $k_{2} \leq k_{1}$ was expected by definitions of the chains $\mathbf{Z}_{\mathbf{1}}$ and $\mathbf{Z}_{\mathbf{2}}$. To see this we note that in the model (5.1) it is possible to change both symbols of the original process $\mathbf{X}$ by the Bernoulli effect $\boldsymbol{\xi}$ whereas in the model (5.2) only the symbol 1 of the process $\mathbf{X}$ has positive probability of being modified by process $\xi$. Since the process $\mathbf{Z}_{3}$ is more general than the other two then the inequality $k_{1} \leq k_{3}$ was also expected.

\section{Acknowledgements}

This is part of the Ph.D. Thesis suported by FAPESP fellowship (grant 2008/10693-5). N. L. Garcia was partially supported by CNPq grants 475504/2008-9, 302755/2010-1 and $476764 / 2010-6$ 


\section{References}

[1] Bühlmann, P., Wyner, A. (1999) Variable length Markov chains.Ann. Statistics, 27(2), pp. $480-513$.

[2] Collet, P., Galves, A., Leonardi, F. (2008), Random Perturbations of Stochastic Processes with Unbounded Variable Length Memory, Eletronic Journal of Probability, 13 (48), pp. 1345-1361.

[3] Galves, A., Maume-Deschamps, V., Schmitt, B. (2008), Exponential inequalities for VLMC empirical trees, ESAIM Prob. Stat., 12, pp. 219-229.

[4] Csiszár, I., Talata, Z. (2006), Context tree estimation for not necessarily finite memory processes, via BIC and MDL, IEEE Trans. Inform. Theory 52(3), pp. 1007-1016.

[5] Dedecker, J., Doukhan, P. (2003), A new covariance inequality and applications, Stochastic Process. Appl., 106(1), pp. 63-80.

[6] Dedecker, J., Prieur, C. (2005), New dependence coefficients. examples and applications to statistics, Probab. Theory Relatated Fields, 132, pp. 203-236.

[7] Duarte, D., Galves, A., Garcia, N. (2006), Markov approximation and consistent estimation of unbounded probabilistic suffix trees, Bull. Braz. Math. Soc. 37(4), pp. $581-592$.

[8] Fernández, R., Galves, A. (2002), Markov approximations of chains of infinite order, Bull. Braz. Math. Soc., 33(3), pp. 1-12.

[9] Fernández, R., Ferrari, P., Galves, A. (2001), Coupling, renewal and perfect simulation of chains of infinite order. Notes for a minicourse at the $V^{\text {th }}$ Brazilian School of Probability, URL: http://www.ime.unicamp.br/ ebp5/.

[10] Ferrari, F. Wyner, A. (2003), Estimation of general stationary processes by variable length Markov chains, Scand. J. Statist., 30(3), pp. 459-480.

[11] Galves, A., Leonardi, F. (2008), Exponential inequalities for empirical unbounded context trees, Progress in Probability, Birkhauser, 60, pp. 257-270.

[12] Galves, A., Löcherbach, E. (2008), Stochastic chains with memory of variable length, TICSP Series, 38, pp. 117-133,

[13] Rissanen, J. (1983), A universal data compression system, IEEE Trans. Inform. Theory, 29(5), pp. 656-664.

[14] Ron, D., Singer, Y. and Tishby, N. (1996), The power of amnesia: Learning probabilistic automata with variable memory length, Machine Learning, 25(2-3), pp. 117-149.

[15] Willems, F. M., Shtarkov, Y. M., Tjalkens, T. J. (1995), The context-tree weighting method: basic properties, IEEE Trans. Inform. Theory, IT-44, pp. 653-664. 